\title{
Coste de las visitas médicas y urgencias asociadas al estrés laboral en España*
}

\author{
M $^{\text {a }}$ LUZ GONZÁLEZ ÁlVAREZ ${ }^{\text {a }}$, CARLOS GAMERO-BURÓN ${ }^{\text {a }}$ \\ a Universidad de Málaga, Facultad de CC.EE., Plaza El Ejido, s/n, 29071 Málaga, España. E-mail: \\ luz@uma.es,gamero@uma.es
}

\section{RESUMEN}

El estrés laboral representa un grave problema para la salud de los trabajadores, las organizaciones y la sociedad. El objetivo de este trabajo es valorar los costes sanitarios que genera debidos al incremento de la utilización de asistencia sanitaria. Con las Encuestas Nacionales de Salud (2006/07 y 2011/12), se han aplicado métodos de propensity score matching y estimación microeconométrica para cuantificar las visitas al médico general, especialista y urgencias asociadas al estrés laboral. La valoración de tales visitas permite concluir que en 2006/07 el coste monetario asciende a 145,26 millones $€$ debidos a las visitas al médico general y a urgencias. En 2011/12, representan 55,17 millones $€$ provocados por las visitas a urgencias hospitalarias.

Palabras clave: Estrés laboral, utilización de asistencia sanitaria, propensity score matching, valoración de costes sanitarios.

\section{Health Care Costs Due to Job Stress in Spain}

\begin{abstract}
Job stress is a serious problem for workers' health, organizations and society. The aim of this paper is to assess the health care costs caused by the increase of health care services. Using the National Health Surveys (2006/07 and 2011/12), we have applied propensity score matching methods and microeconometric estimation in order to quantify the visits to general practitioner (GP), specialists and emergency visits due to job stress. In 2006/07 the assessment of these visits permits to conclude that the economic costs are 145.26 million $€$ due to GP visits and emergency rooms. In 2011/12 the costs are 55.17 million $€$ due to hospital emergencies.
\end{abstract}

Keywords: Job Stress, Health Care Utilisation, Propensity Score Matching, Health Care Costs Assessment.

Clasificación JEL: J22, J28, I12, C25

\footnotetext{
${ }^{*}$ Los autores agradecen los comentarios realizados por los dos evaluadores anónimos, los cuales han permitido mejorar este trabajo. Las opiniones expresadas son las de los autores y no comprometen a la institución a la que pertenecen. Los autores también agradecen la financiación recibida de la Cátedra de Economía de la Salud y Uso Racional del Medicamento de la Universidad de Málaga y del proyecto de excelencia P09SEJ4859 de la Junta de Andalucía.
}

Artículo recibido en junio de 2013 y aceptado en agosto de 2013

Artículo disponible en versión electrónica en la página www.revista-eea.net, ref. ə-31214 


\section{INTRODUCCIÓN}

Las condiciones económicas actuales están provocando la puesta en marcha de nuevas prácticas laborales, destinadas a mejorar la productividad y la calidad, aumentando, por tanto, las posibilidades de que surjan nuevos riesgos para la salud y la seguridad o que algunos de los peligros existentes se vuelvan mayores. A estas nuevas prácticas se suma el efecto de los cambios en la demografía laboral, con una mayor proporción de empleados mayores en la mano de obra europea. En España, según la Encuesta de Población Activa, el porcentaje de población ocupada mayor de 55 años ha aumentado de un 11,1\% en el primer trimestre de 2005 a un 14,3\% en el primer trimestre de 2013. Este incremento ha sido mayor en el caso de las mujeres, ya que representaban un 3,5\% de los ocupados en 2005 y en la actualidad suponen el 6,1\%. Estos cambios conducen a los riesgos psicosociales emergentes, que se definen como "los aspectos del diseño, organización y gestión del trabajo así como del contexto social y medioambiental, que pueden causar daño psicológico, social o físico a los trabajadores” (EASHW, 2007).

Entre los denominados "riesgos emergentes", un lugar especialmente destacado lo ocupan los "riesgos de origen psicosocial” y, en particular, el estrés laboral, al ser considerado como uno de los principales desafíos para la seguridad y la salud laboral, los salarios, o el absentismo (EC-DGESA, 1999; French y Dunlap, 1998; Groot y Maasen van den Brink, 1999; Leontaridi y Ward, 2002). En la creciente literatura económica sobre los determinantes de la satisfacción laboral, bienestar individual y felicidad, son escasos los estudios que han investigado el estrés laboral en España desde una perspectiva económica. Los motivos pueden estar relacionados con que es un problema no muy visible y tienden a subestimarse sus efectos tanto para las empresas, como para la economía en general, ya que una parte de los cuales son intangibles. Por ejemplo, las bajas laborales son obvias, pero los efectos a largo plazo no se perciben fácilmente y otros como la reducción de la creatividad y la innovación son más difíciles de medir. Por otro lado, son muy pocas las bases de datos representativas a nivel nacional que contienen información sobre estrés laboral.

Los estudios de Gamero (2010a, 2010b) investigan los factores explicativos del estrés laboral soportado por la población asalariada española y sitúan la factura por jornadas laborales perdidas atribuibles a esta dolencia para el año 2004 en el 0,11\% del PIB. El trabajo de García-Gómez et al., (2012) estudia los costes laborales de las enfermedades mentales atribuibles al trabajo en España para 2010, dedicando un apartado al estrés, y centrándose en el coste de la incapacidad temporal, la duración de la baja y las pérdidas de productividad.

La carencia de trabajos que relacionen el estrés con la utilización de servicios sanitarios, particularmente en España, es aún más notoria. Y es que la única base de datos que contiene amplia información sobre ambos aspectos es la En- 
cuesta Nacional de Salud de 2006/07 y la de 2011/12. El interés por evaluar los costes del estrés laboral, en términos sanitarios, reside en que a menudo las empresas no soportan los costes completos de los accidentes profesionales, dolencias, lesiones profesionales o enfermedades relacionadas con el trabajo y tienen que ser soportados por el Sistema de Seguridad Social y el Sistema Nacional de Salud del país.

Inmersa en este contexto, la investigación que aquí se presenta constituye un reto, ya que persigue como objetivo fundamental evaluar, con el auxilio de técnicas microeconométricas, los costes sanitarios que resultan como consecuencia de la previsible mayor utilización de asistencia sanitaria por parte de los trabajadores con mayor nivel de estrés laboral.

Su contenido se estructura como sigue. La sección 2 expone métodos susceptibles de aplicación para explicar la utilización de servicios sanitarios y su posible relación con el estrés laboral. En la sección 3 se realiza una descripción de la base de datos utilizada y un análisis descriptivo de la prevalencia del estrés y su relación con la utilización de servicios sanitarios en España. La sección 4 presenta los resultados de la estimación de los modelos microeconométricos explicativos del número de consultas demandadas por los trabajadores y la cuantificación del comportamiento diferencial que presentan los trabajadores sometidos a estrés. En la sección 5 se procede a convertir en términos monetarios dicho comportamiento diferencial, aplicando los precios públicos de los servicios sanitarios prestados a terceros obligados al pago en centros sanitarios dependientes de los distintos Sistemas Regionales de Salud. Por último, la sección 6 recoge las principales conclusiones que se derivan de esta investigación.

\section{ESTRÉS LABORAL Y UTILIZACIÓN DE SERVICIOS SANITARIOS}

El estrés laboral es un fenómeno complejo, pero a día de hoy está generalmente aceptado que se contempla como el desequilibrio percibido entre las demandas internas y externas que enfrenta el individuo y la habilidad percibida para afrontar la situación (Di Martino, 1992; Cox, 1993). Según la teoría dominante de estrés laboral de Karasek y Theorell (1990), los puestos de trabajo que combinan una elevada demanda psicológica y un reducido poder de decisión fomentan los problemas de salud física y mental.

El estudio del estrés ocupacional y sus consecuencias para la salud constituye un área importante de la investigación en las ciencias del comportamiento. La literatura médica ofrece numerosos estudios de problemas físicos y psicológicos relacionados con el estrés (MacFadyen et al., 1996), tales como enfermedades cardiovasculares (Rosengren et al., 2004; Chandola et al., 2008), infecciones respiratorias (Turner-Cobb y Steptoe, 1996), traumatológicos, migrañas (Sorbi et al., 1996; Benedettis y Lorenzetti, 1992) e inmunológicas 
(Brosschot et al., 1994; Kramer et al., 2000). Sin embargo, tales medidas fisiológicas son problemáticas, puesto que una larga serie de factores distintos de los relacionados con el estrés pueden influir en la salud.

Por lo que respecta a los servicios sanitarios, los enfoques teóricos susceptibles de aplicación al análisis de la demanda y utilización de los mismos son dos: 1) la teoría tradicional del consumidor, considerando al individuo como el principal agente para determinar la demanda de servicios sanitarios, aunque condicionado por la organización del sistema sanitario (Grossman, 1972a, b), y 2) los modelos principal-agente, en los que el médico, como agente del paciente, determina la cantidad de servicios médicos utilizados en nombre del paciente (principal) una vez que se ha producido la primera visita (Zweifel, 1981).

El indicador por excelencia de la utilización de asistencia sanitaria suele ser el número de consultas médicas en un determinado período. Dado que esta variable sólo toma valores enteros positivos, desde la publicación del trabajo de Cameron et al., (1988) que analizaba utilización y seguro médico, los trabajos empíricos estiman modelos microeconométricos count data o de recuento ${ }^{1}$. La especificación del modelo explicativo de utilización médica responde a la siguiente formulación:

$$
E\left(U_{i} \mid X_{k i}\right)=e^{\gamma_{1} X_{k i}+\xi_{i}}
$$

Las conclusiones de tales trabajos confirman que los factores explicativos más relevantes para explicar la utilización de servicios sanitarios $\mathrm{U}_{\mathrm{i}}$ son: el estado de salud, aproximado mediante valoraciones subjetivas u otras medidas objetivas (indicadores fisiológicos, diagnósticos clínicos,...); las características demográficas de la población; determinados condicionantes socioeconómicos (renta, nivel educativo u ocupación); o la región de residencia. Los cambios sociales y de estilos de vida son aspectos a los que se les presta mayor atención en los últimos estudios sobre demanda de salud y, por ende, de servicios sanitarios. Es obvia la importancia de comportamientos antisaludables como el tabaco, el alcohol, la obesidad o los riesgos psicosociales, entre los que se incluye al estrés, acentuada por factores tales como la creciente incorporación de la mujer al mercado de trabajo o la vivencia de una crisis económica como la actual. Sin embargo, a la asociación entre utilización de asistencia médica y estrés se le ha dedicado escasa atención, a pesar de las recomendaciones de algunos trabajos pioneros (Mechanic, 1979; Gortmaker et al., 1982).

Uno de los pocos trabajos existentes es el de Azagba y Sharaf (2011), empleando como fuente de información la Encuesta Nacional de Salud de Canadá.

\footnotetext{
${ }^{1}$ En España, uno de los primeros trabajos, aunque referido únicamente a las visitas al médico especialista en Cataluña es de Vera-Hernández (1999). Urbanos (2000) lo amplia a todo el sistema sanitario público español, analizando visitas a médicos generales, especialistas, servicios de urgencias y días de estancia en el hospital.
} 
Sus conclusiones más relevantes son que los trabajadores con niveles de estrés elevados aumentan las visitas al médico general y al especialista, sin embargo, cuando van incorporando al modelo factores tan importantes como el estado de salud, o tener problemas crónicos, dicha influencia se va reduciendo hasta representar el 10 y 14 por ciento, en el caso del médico general y del especialista, respectivamente. El problema de este planteamiento está en atribuir todo el efecto causal del estrés sobre la utilización al valor del coeficiente estimado, ajustando por otras variables, debido a la elevada correlación existente entre algunas de las variables explicativas.

La forma de corregir el posible sesgo de tales estimaciones se basa en una nueva corriente de investigación que adapta los diseños experimentales a bases de datos observacionales. La idea es comparar, como en los ensayos clínicos, dos grupos de pacientes similares (grupo tratamiento y grupo control) que únicamente se diferencian en el tratamiento $(T)$, con objeto de cuantificar los verdaderos efectos del citado tratamiento.

Suponiendo que $T$ toma dos valores 1- tratamiento (estresados) y 0 - ausencia de tratamiento, $U_{i 1}$ y $U_{i 0}$ representan la utilización del individuo $i$ con y sin tratamiento, respectivamente. Para cada individuo $i$, el efecto del tratamiento vendría dado por la diferencia $U_{i 1}-U_{i 0}$, sin embargo, con los datos procedentes de una encuesta sólo tendremos información de un individuo en uno de los grupos o $U_{i 1}$ (estresados) o $U_{i 0}$ (no estresados). Si bien es posible estimar el efecto medio del tratamiento sobre los tratados (ATT):

$$
A T T=E\left(U_{1}-U_{0} \mid T=1\right)
$$

que mide, por término medio, cuánto varía la utilización para aquellos individuos que se someten al tratamiento, es decir, los que están estresados.

El método de evaluación no experimental, denominado propensity score matching (PSM), estima los efectos del tratamiento utilizando la información de un grupo de individuos del grupo control (no estresados), pero que tienen características observables similares a los del grupo de tratamiento (estresados), con objeto de identificar lo que le habría ocurrido a las unidades participantes en ausencia de la intervención. La clave es buscar y construir un grupo de comparación válido (contrafactual) para calcular la diferencia de los resultados entre participantes y no participantes que son similares desde el punto de vista observacional.

Uno de los temas críticos en la aplicación de técnicas de matching o emparejamiento, para eliminar el sesgo potencial, es definir claramente y justificar lo que significa "similar", sobre todo, cuando se trabaja con múltiples variables explicativas. Rosenbaum y Rubin (1983) resolvieron este problema proponiendo el cálculo del propensity score. Éste mide la probabilidad estimada de que un individuo de la muestra participe en el grupo de tratamiento, dado un 
conjunto $X$ de variables observables. De esta forma, el propensity score resumirá toda la información relevante contenida en las variables $X$ y permitirá emparejar individuos que experimentan estrés laboral con otros idénticos del grupo de control en función únicamente del propensity score, en lugar de un conjunto completo de variables observadas $X$.

En ocasiones, los criterios que determinan la participación en el grupo de tratamiento (estresados) son desconocidos, por lo que es conveniente estimar modelos logit o probit controlando por todas las variables que supuestamente influyen en la probabilidad de pertenecer al grupo de tratamiento.

Los primeros trabajos que aplican métodos de propensity score matching para explicar como la salud incide en el comportamiento de los trabajadores respecto al mercado de trabajo corresponden a Lechner y Vázquez-Álvarez (2004), Frolich et al., (2004), Dano (2005), García-Gómez y López Nicolás (2006) y García-Gómez (2011).

El único inconveniente de esta metodología reciente está en que se ha aplicado para estimar cómo varía una variable continua o una binaria entre dos grupos de individuos, pero éste no es el caso si la utilización de servicios sanitarios se cuantifica por el número de visitas, por tal razón en este trabajo se propone hacer una combinación de las dos metodologías. En primer lugar, seleccionar muestras de estresados y no estresados que sean similares en cuanto al resto de variables explicativas, a través del propensity score, para posteriormente estimar modelos count data -binomial negativo- y cuantificar el efecto del estrés sobre la utilización de consultas médicas.

\section{FUENTE DE INFORMACIÓN Y ANÁLISIS DESCRIPTIVO}

La opción más simple de medir el estrés laboral está basada en la propia declaración del individuo sobre el nivel percibido de tensión psicológica o mental en el trabajo. Tales valoraciones subjetivas han sido aceptadas en la literatura económica hasta la fecha como medio de predecir y entender el comportamiento individual en el mercado de trabajo ${ }^{2}$.

Para realizar el ejercicio de evaluación perseguido con esta investigación se ha seleccionado como fuente de información la Encuesta Nacional de Salud (ENS), correspondiente a los años 2006/07 y 2011/12³. El ámbito geográfico de

\footnotetext{
${ }^{2}$ Véase Freeman (1978). Este tipo de medidas no están, sin embargo, exentas de problemas. El principal, probablemente, sea el sesgo de deseabilidad social en las respuestas: los trabajadores podrían estar inclinados a indicar que experimentan estrés en la medida en que esto implica que el trabajador es un miembro valioso de la organización.

${ }^{3}$ La Encuesta de Calidad de Vida en el Trabajo (ECVT) también es una base de datos representativa a nivel nacional de la población ocupada en España. Incluye en su cuestionario una medida de estrés autopercibido y otras cuestiones sobre el puesto de trabajo, pero no recoge preguntas sobre el estado de salud y la frecuencia con la que se utilizan determinados servicios sanitarios.
} 
esta encuesta es todo el territorio nacional, siendo la población de estudio en 2006 los adultos de 16 y más años residentes en hogares familiares. La referida a 2011/12 incluye a los adultos de 15, con objeto de homogeneizar los criterios de población con otras encuestas de salud a nivel europeo.

Las muestras inicialmente seleccionadas están compuestas por individuos que en el momento de la entrevista están ocupados con edades comprendidas entre los 16 (15) y 64 años, ambos inclusive. La encuesta de 2011/12 incluye en esta categoría a las personas que estuvieran ausentes de su trabajo por estar de baja. La pregunta sobre estrés laboral que incluyen ambos cuestionarios de la ENS es la siguiente: Globalmente, $\mathrm{y}$ teniendo en cuenta las condiciones en que realiza su trabajo, indique cómo considera Vd. el nivel de estrés de su trabajo, según una escala de 1 (nada estresante) a 7 (muy estresante).

Los datos recogidos en la Tabla 1 muestran la distribución porcentual de las respuestas sobre el estrés para dar una idea de la incidencia del problema entre la población ocupada española durante los períodos analizados. Las cifras se han obtenido teniendo en cuenta el diseño muestral de la encuesta y las ponderaciones. El porcentaje de trabajadores que declaran padecer mayor nivel de estrés laboral ha aumentado en los cinco años transcurridos, coincidiendo con la preocupación por las condiciones de trabajo y la incertidumbre a perder el empleo.

Tabla 1

Distribución porcentual del estrés laboral

\begin{tabular}{|c|c|c|}
\hline Escala & $\mathbf{2 0 0 6 / 0 7}$ & $\mathbf{2 0 1 1 / 1 2}$ \\
\hline 1 & 9,124 & 9,013 \\
\hline 2 & 8,964 & 9,221 \\
\hline 3 & 13,39 & 11,64 \\
\hline 4 & 23,11 & 18,20 \\
\hline 5 & 23,40 & 24,91 \\
\hline 6 & 12,99 & 16,91 \\
\hline 7 & 9,032 & 10,11 \\
\hline Media & 4,178 & 4,319 \\
\hline$N$ & 13077 & 8541 \\
\hline
\end{tabular}

Fuente: Elaboración propia a partir de ENS 2006/07 y 2011/12.

En cuanto a la utilización de asistencia sanitaria, en este trabajo sólo se van a cuantificar los relativos a tres servicios médicos: el número de consultas al médico general en las últimas cuatro semanas; el número de consultas al médico especialista en el mismo período; y el número de visitas a urgencias en el último año. Una vez eliminadas las observaciones missing de estrés laboral y utiliza- 
ción de asistencia sanitaria, la muestra de 2006/07 es de 13.077 individuos y la de 2011/12 está formada por 8.541 trabajadores, que representan a 18.429 .970 y 16.913.153 de ocupados, respectivamente.

La Tabla 2 ofrece en términos puramente descriptivos la relación entre el estrés laboral y la demanda de los tres servicios sanitarios. Por un lado, se observa en relación con el periodo de tiempo considerado que la utilización del médico general es mayor que la del especialista. Aunque los valores medios son mayores en el caso de las urgencias, hay que considerar que miden el número de visitas a lo largo de un año. En general, se aprecia una disminución de la utilización media del médico general en el tiempo, salvo para los que declaran la escala 2. Se observa un aumento de las visitas medias al especialista para los niveles centrales de estrés, y un descenso para los que declaran menores y mayores nivel de estrés. Y, por último, una disminución de la utilización media de los servicios de urgencia, excepto para las personas que declaran una escala igual a 5, cuyo valor medio se mantiene.

Tabla 2

Número medio de consultas médicas según nivel de estrés laboral

\begin{tabular}{|c|c|c|c|c|c|c|}
\hline & \multicolumn{2}{|c|}{ M. General } & \multicolumn{2}{c|}{ M. Especialista } & \multicolumn{2}{c|}{ M. Urgencias } \\
\hline Escala & $\mathbf{2 0 0 6 / 0 7}$ & $\mathbf{2 0 1 1 / 1 2}$ & $\mathbf{2 0 0 6 / 0 7}$ & $\mathbf{2 0 1 1 / 1 2}$ & $\mathbf{2 0 0 6 / 0 7}$ & $\mathbf{2 0 1 1 / 1 2}$ \\
\hline 1 & 0,3016 & 0,2591 & 0,1387 & 0,1165 & 0,4560 & 0,2973 \\
\hline 2 & 0,2423 & 0,2548 & 0,1655 & 0,1457 & 0,4334 & 0,3147 \\
\hline 3 & 0,3137 & 0,2550 & 0,1283 & 0,1652 & 0,4285 & 0,3705 \\
\hline 4 & 0,2864 & 0,2452 & 0,1513 & 0,1696 & 0,4177 & 0,3820 \\
\hline 5 & 0,3037 & 0,2627 & 0,1875 & 0,1891 & 0,3729 & 0,3733 \\
\hline 6 & 0,3161 & 0,2585 & 0,1925 & 0,1772 & 0,4957 & 0,4819 \\
\hline 7 & 0,4522 & 0,3586 & 0,2592 & 0,2361 & 0,6853 & 0,5356 \\
\hline
\end{tabular}

Fuente: Elaboración propia a partir de ENS 2006/07 y 2011/12.

Para definir un grupo de estresados homogéneo para los tres servicios y en los dos periodos, en primer lugar, se aplicaron contrastes basados en la t-de student para diferencias de la utilización media con distintas agrupaciones de estresados y, en segundo lugar, se estimaron modelos de regresión count databinomial negativo de la utilización de asistencia sanitaria en función únicamente de variables binarias representativas de cada nivel de estrés laboral.

En todos los casos, este análisis inicial mostraba que el grupo de población formado por aquellos que declaran un nivel de estrés 7 y el grupo integrado por los que perciben un nivel de estrés 6 o 7, presentaban un comportamiento diferenciador en la utilización respecto al resto de trabajadores. En algunos casos, la categoría 5 resultaba significativa al $10 \%$, mostrando un comportamiento más 
similar al de trabajadores con menores niveles de estrés. La sospecha era que, al considerar otras características explicativas de la utilización, esa diferencia se anularía. Además, el grupo de trabajadores que declaran el nivel 5 es el más numeroso en las dos encuestas, en torno al $25 \%$ de la población, por lo que si se uniese al grupo 6 y 7 estaríamos comparando dos muestras con la mitad de las observaciones en cada grupo. Por estas razones, se ha creído que lo más apropiado era considerar, para todo el análisis posterior, como trabajadores estresados a aquellos que manifiestan el nivel 6 o 7 (muy estresante). A pesar de ello, todo el análisis empírico también se ha realizado para el grupo de estrés 5-6-7, con objeto de comprobar la robustez de los resultados.

El uso de datos observacionales para analizar el efecto causal del estrés sobre la utilización de asistencia sanitaria puede sesgar las estimaciones, sobre todo, si los sujetos sometidos a estrés difieren sustancialmente de los trabajadores que no lo están. Por ello, en la Tabla 3 se recogen las variables representativas de utilización de asistencia sanitaria y de todas aquellas que se ha considerado pueden afectar a ésta y estén relacionadas con el nivel de estrés laboral, así como el resultado de haber aplicado contrastes de diferencias de medias $\left(\bar{X}_{E}-\bar{X}_{N E}\right)$ de todas las variables explicativas en el grupo de estresados (E) y no estresados (NE).

Por lo que respecta al estado de salud, se ha definido mediante 1) salud autopercibida, representada por dos variables binarias (muy buena salud y buena salud frente a regular, mala y muy mala); 2) otra variable binaria indica si el trabajador declara padecer alguna de las enfermedades crónicas o de larga duración que recoge la ENS; 3) otra recoge si el trabajador ha sufrido un accidente; $y, 4)$ la última informa sobre si ha visto limitada su actividad habitual en los últimos 15 días debido a problemas de huesos, depresión, gripe, dolor de cabeza, problemas digestivos, alergias, angustia, cansancio, etc., que pueden explicar la utilización del médico general y del especialista al estar referida sólo a las últimas cuatro semanas.

En cuanto a hábitos de vida, se ha tenido en cuenta si el individuo: 1) se declara fumador, ya sea a diario o no diariamente; 2) toma alcohol, si declara una frecuencia de consumo diaria o semanalmente; 3) realiza alguna actividad física en su tiempo libre y 4) las horas que duerme habitualmente al día. Este conjunto de variables se ha considerado por su influencia sobre la salud y la utilización de servicios sanitarios. El problema es que también puede presentar una posible endogeneidad o asociación con el estrés laboral, en el sentido de que el estrés puede provocar hábitos perjudiciales para la salud. Cooper et al., (1990) obtienen que el impacto de factores estresantes del trabajo sobre medidas relacionadas con el alcohol es pequeña, incluso para los individuos más vulnerables, si bien estos individuos no son muy representativos en una muestra de ocupados. Schmidt et al., (2010) concluyen, en contra de lo esperado, que los 
factores del estrés ocupacional están asociados con menores niveles de dependencia de la nicotina. Akerstedt et al., (2002) sostienen que el esfuerzo físico o puestos con una elevada demanda de trabajo son indicadores de riesgo para sufrir trastornos del sueño, sin embargo, el factor más importante era la incapacidad para dejar de pensar en el trabajo durante el tiempo de ocio. Kouvonen et al., (2013) disponen de datos longitudinales para evaluar que un incremento en las condiciones de estrés en el lugar de trabajo estaba débilmente asociado con el aumento de una insuficiente actividad física, incluso ante una exposición repetida a tales factores estresantes. Los propios autores no excluyen la posibilidad de causalidad inversa, es decir, los trabajadores pueden experimentar más estrés en el trabajo por la falta de actividad física.

Aunque haya que tener presente estos problemas, en este trabajo el estrés se basa en una percepción del trabajador y no en condiciones estresantes objetivas del puesto de trabajo. La ENS no ofrece tanta información sobre las condiciones y características de dicho puesto de trabajo. Con respecto al alcohol y el tabaco, no se han obtenido diferencias de medias significativas y las varianzas son muy similares entre estresados y no estresados, ya que no se mide la cantidad consumida de cada producto, sino la posibilidad de que fume a diario o semanalmente, o que consuma alcohol a diario o semanalmente. Por lo que respecta al sueño, no se puede medir la calidad del mismo, sino la cantidad de horas que duerme al día. Una menor cantidad no puede ser sinónimo de que el estrés le afecte en mayor medida, porque las necesidades de descanso de cada persona pueden ser muy dispares. En cualquier caso, el objetivo no es establecer relaciones de causalidad, sino obtener muestras de trabajadores similares en todas las características que afectan a la utilización de asistencia sanitaria.

Entre las características personales y familiares se incluyen: 1) indicadores de sexo y edad; 2) estado civil (casado, separado o divorciado y viudo frente a solteros); y 3) las responsabilidades familiares se han recogido mediante ocho variables dicotómicas: ser el miembro del hogar que más aporta al presupuesto; ser la única persona del hogar que genera ingresos; ser padre o madre solo con algún hijo menor de 25 años; ser el único que se ocupa de discapacitados del hogar; compartir su cuidado con otras personas del hogar; tener hijos menores de 15 años; ser el único que se ocupa del cuidado de los menores del hogar; y, compartir su cuidado con otras personas del hogar. En cuanto a factores socioeconómicos, no se han incluido intervalos de renta, puesto que el INE no los ha incorporado aún a la base de microdatos de la ENS 2011/12. El nivel educativo del trabajador que se ha considerado ha sido: bachillerato, FP superior y estudios universitarios, es decir, los estudios no obligatorios. Por lo que respecta al empleo, se han seleccionado indicadores de: 1) tipo de relación laboral (los que tienen un contrato inferior a dos años, contrato indefinido y funcionarios frente a los que trabajan por cuenta propia); 2) tipo de jornada laboral (partida, reducida, por turnos, irregular u otro tipo frente a jornada continua); 3) estar muy 
satisfecho laboralmente (en una escala de 1 a 7, declarar una escala 6 o 7); 4) tener reconocimiento por el trabajo desarrollado; y 5) tener trabajadores a cargo (5-10 trab., 10-20 trab., > 20 trab. frente a menos de 5 trab.). Por último, se incluyen indicadores de la Comunidad Autónoma en la que reside el trabajador (Referencia: Extremadura).

Las diferencias en la utilización de los tres servicios son significativas en los dos años analizados, se trata ahora de comprobar si tales divergencias son debidas a otras características. También se ha calculado el porcentaje de sesgo estandarizado para comprobar la disparidad entre estresados y no estresados en relación con las variables explicativas enumeradas anteriormente. Considerando los mayores porcentajes de sesgo en valor absoluto $(10<\text { sesgo }<25)^{4}$, las variables que no están equilibradas en ambos grupos de trabajadores difieren según el año de la encuesta (9 - 2006/07 y 6 - 2011/12). Declarar una enfermedad crónica, tener alguna limitación de la actividad habitual, las horas de sueño y tener un contrato de trabajo inferior a dos años son comunes en los dos periodos. En 2006/07, tampoco están equilibradas las variables: declarar un buen estado de salud, ser la persona que más ingresos aporta, estar casado, estar muy satisfecho con su trabajo y obtener reconocimiento por el mismo. Y en 2011/12, residir en dos CCAA (Castilla-Mancha y Cataluña).

\section{Tabla 3}

Diferencias de medias según nivel de estrés y \% sesgo estandarizado

\begin{tabular}{|c|c|c|c|c|c|c|}
\hline \multirow[b]{2}{*}{ Variable } & \multicolumn{3}{|c|}{$2006 / 07$} & \multicolumn{3}{|c|}{$2011 / 12$} \\
\hline & $\bar{X}_{E}-\bar{X}_{N E}$ & & $\%$ Sesgo & $\bar{X}_{E}-\bar{X}_{N E}$ & & $\%$ Sesgo \\
\hline Visitas M. General (mes): & 0,07896 & 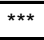 & & 0,04028 & ** & \\
\hline Visitas M. Especialista (mes): & 0,06150 & 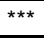 & & 0,03331 & ** & \\
\hline Visitas M. Urgencias (12 meses): & 0,16113 & *** & & 0,14379 & $\star \star \star *$ & \\
\hline \multicolumn{7}{|l|}{ Estado de salud: } \\
\hline Muy bueno & $-0,0255$ & $* *$ & $-6,3098$ & $-0,0316$ & $\star \star$ & $-7,5727$ \\
\hline Bueno & $-0,0606$ & 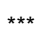 & $-12,0541$ & $-0,0201$ & & $-4,1042$ \\
\hline Enfermedad crónica & 0,0756 & $\star * \star$ & 16,3729 & 0,0749 & $* * *$ & 15,8934 \\
\hline $\begin{array}{l}\text { Limitación Activ. Habitual } \\
\text { (últimos } 15 \text { días) }\end{array}$ & 0,0565 & $\star * \star$ & 15,0934 & 0,0749 & $\star \star \star \star ~$ & 15,8934 \\
\hline Accidente & 0,0244 & $\star \star \star *$ & 7,2781 & 0,0070 & & 2,4347 \\
\hline \multicolumn{7}{|l|}{ Hábitos: } \\
\hline Fumador & 0,0023 & & 0,4701 & 0,0169 & & 3,6201 \\
\hline Alcohol & $-0,0018$ & & $-0,3672$ & $-0,0167$ & & $-3,4013$ \\
\hline Horas sueño & $-0,3351$ & 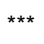 & $-27,1180$ & $-0,1809$ & 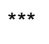 & $-15,4456$ \\
\hline Ejercicio físico & $-0,0383$ & *** & $-7,6434$ & $-0,0447$ & $\star \star \star \star$ & $-9,0715$ \\
\hline
\end{tabular}

${ }^{4}$ Criterio basado en los resultados de Cochran y Rubin (1973). 
Tabla 3 (continuación)

Diferencias de medias según nivel de estrés y \% sesgo estandarizado

\begin{tabular}{|c|c|c|c|c|c|c|}
\hline \multirow[b]{2}{*}{ Variable } & \multicolumn{3}{|c|}{$2006 / 07$} & \multicolumn{3}{|c|}{$2011 / 12$} \\
\hline & $\bar{X}_{E}-\bar{X}_{N E}$ & & $\%$ Sesgo & $\bar{X}_{E}-\bar{X}_{N E}$ & & $\%$ Sesgo \\
\hline \multicolumn{7}{|l|}{ Características personales: } \\
\hline Edad & 0,8612 & *** & 8,3225 & 0,2084 & & 2,0789 \\
\hline Mujer & 0,0089 & & 1,8050 & 0,0213 & & 4,3192 \\
\hline \multicolumn{7}{|l|}{ Responsabilidades familiares } \\
\hline Aporta más ingresos & 0,0594 & $\star * *$ & 12,0409 & 0,0283 & $\star *$ & 5,9059 \\
\hline Único miembro genera ingresos & 0,0172 & & 3,4516 & $-0,0073$ & & $-1,5446$ \\
\hline Casado & 0,0530 & $* * *$ & 10,8693 & 0,0130 & & 2,7088 \\
\hline Viudo & 0,0020 & & 1,8897 & 0,0021 & & 1,8826 \\
\hline Separado o Divorciado & 0,0192 & $\star \star \star *$ & 7,6796 & 0,0151 & $* * \star$ & 5,9633 \\
\hline $\begin{array}{l}\text { Padre o madre solo con hijos } \\
\text { menores de } 25 \text { años }\end{array}$ & 0,0018 & & 1,2000 & 0,0081 & & 4,1820 \\
\hline Hogar con menores de 15 años & 0,0348 & $\star \star * *$ & 7,4734 & 0,0006 & & 0,1350 \\
\hline Cuida solo de menores 15 años & $-0,0002$ & & $-0,1200$ & $-0,0042$ & & $-2,0476$ \\
\hline $\begin{array}{l}\text { Comparte cuidado de menores } \\
\text { de } 15 \text { años }\end{array}$ & 0,0237 & $\star \star$ & 6,2382 & 0,0099 & & 2,2903 \\
\hline Cuida solo de discapacitados & 0,0015 & & 1,9494 & 0,0020 & & 1,9660 \\
\hline Comparte cuidado de discap. & 0,0001 & & 0,1125 & 0,0021 & & 1,9254 \\
\hline \multicolumn{7}{|l|}{ Nivel educativo: } \\
\hline Educ. Secund. $2^{\mathrm{a}}$ etapa & $-0,0216$ & $\star *$ & $-5,8261$ & $-0,0133$ & & $-3,9255$ \\
\hline FP superior & $-0,0009$ & & $-0,3073$ & 0,0045 & & 1,5043 \\
\hline Universitario & 0,0319 & $\star * \star$ & 7,1619 & 0,0387 & $\star \star \star *$ & 8,7247 \\
\hline \multicolumn{7}{|l|}{ Relación laboral: } \\
\hline Funcionario & 0,0077 & & 2,7000 & $-0,0073$ & & $-2,8221$ \\
\hline Indefinido & 0,0434 & $\star \star \star *$ & 8,6335 & 0,0330 & $\star \star$ & 6,7324 \\
\hline Contrato & $-0,0836$ & 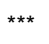 & $-20,4778$ & $-0,0433$ & 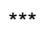 & $-11,3819$ \\
\hline \multicolumn{7}{|l|}{ Resposabilidades: } \\
\hline Muy satisfecho trabajo & 0,0540 & $* * *$ & 10,7911 & 0,0209 & $* \star \star$ & 4,3135 \\
\hline Reconocimiento trabajo & $-0,0733$ & 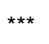 & $-14,6684$ & $-0,0440$ & $\star * *$ & $-9,1283$ \\
\hline Responsable de 5 a $10 \mathrm{tr}$ & 0,0204 & *** & 9,8052 & 0,0152 & 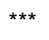 & 7,7592 \\
\hline Responsable de 11 a $20 \mathrm{tr}$ & 0,0118 & $\star * \star$ & 7,7605 & 0,0088 & ** & 6,1109 \\
\hline Responsable de más 20 tr. & 0,0130 & $* * *$ & 7,8859 & 0,0130 & 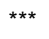 & 8,1666 \\
\hline
\end{tabular}


Tabla 3 (continuación)

Diferencias de medias según nivel de estrés y \% sesgo estandarizado

\begin{tabular}{|c|c|c|c|c|c|c|}
\hline \multirow[b]{2}{*}{ Variable } & \multicolumn{3}{|c|}{$2006 / 07$} & \multicolumn{3}{|c|}{ 2011/12 } \\
\hline & $\bar{X}_{E}-\bar{X}_{N E}$ & & $\%$ Sesgo & $\bar{X}_{E}-\bar{X}_{N E}$ & & $\%$ Sesgo \\
\hline \multicolumn{7}{|l|}{ Jornada laboral: } \\
\hline J. partida & 0,0047 & & 0,9292 & 0,0209 & $* \star *$ & 4,3135 \\
\hline J. reducida & $-0,0074$ & * & $-4,7664$ & $-0,0440$ & $* \star *$ & $-9,1283$ \\
\hline J. turnos & 0,0192 & $\star *$ & 5,9972 & 0,0152 & $\star * \star$ & 7,7592 \\
\hline J. irregular o variable & 0,0178 & $\star *$ & 5,2113 & 0,0088 & ** & 6,1109 \\
\hline J. otro tipo & 0,0008 & & 0,6944 & 0,0130 & $* * *$ & 8,1666 \\
\hline \multicolumn{7}{|l|}{ Comunidad Autónoma: } \\
\hline Andalucía & 0,0090 & & 2,4235 & 0,0204 & * & 5,5332 \\
\hline Aragón & $-0,0015$ & & $-0,8678$ & $-0,0036$ & & $-2,1680$ \\
\hline Asturias & $-0,0056$ & $\star \star$ & $-4,2199$ & $-0,0067$ & ** & $-5,3073$ \\
\hline Baleares & 0,0025 & & 1,4998 & $-0,0043$ & & $-2,8544$ \\
\hline Canarias & 0,0075 & & 3,4245 & 0,0031 & & 1,6052 \\
\hline Cantabria & 0,0007 & & 0,5752 & $-0,0025$ & & $-2,4671$ \\
\hline Castilla León & $-0,0014$ & & $-0,6235$ & $-0,0172$ & $* * *$ & $-8,7510$ \\
\hline Castilla Mancha & $-0,0036$ & & $-1,8445$ & $-0,0221$ & $\star \star \star *$ & $-14,2925$ \\
\hline Cataluña & $-0,0030$ & & $-0,8184$ & 0,0681 & *** & 16,5424 \\
\hline Com. Valenciana & $-0,0010$ & & $-0,3239$ & $-0,0217$ & ** & $-7,6696$ \\
\hline Galicia & $-0,0063$ & & $-2,7160$ & $-0,0084$ & & $-3,8177$ \\
\hline Madrid & 0,0025 & & 0,6858 & 0,0241 & * & 6,4558 \\
\hline Murcia & 0,0051 & & 2,7716 & 0,0049 & & 2,7210 \\
\hline Navarra & $-0,0051$ & $\star \star \star *$ & $-4,7874$ & $-0,0090$ & $* * *$ & $-9,6557$ \\
\hline País Vasco & $-0,0019$ & & $-0,8469$ & $-0,0110$ & $* *$ & $-5,5439$ \\
\hline Rioja & 0,0014 & & 1,5063 & $-0,0026$ & ** & $-3,3751$ \\
\hline Ceuta y Melilla & 0,0015 & $* * *$ & 2,5533 & $-0,0005$ & & $-1,1717$ \\
\hline
\end{tabular}

Fuente: Elaboración propia a partir de ENS 2006/07 y 2011/12.

\section{RESULTADOS EMPÍRICOS DE LA UTILIZACIÓN DE ASISTENCIA SANITARIA ASOCIADA AL ESTRÉS LABORAL}

El propensity score estima la probabilidad de estar muy estresado en función de todas las variables que afectan a la utilización de asistencia sanitaria y al estrés mediante un modelo de regresión logit. En función de dicha probabilidad, el método propensity score matching empareja individuos del grupo de estresa- 
dos con aquellos otros del grupo control que tengan características similares en las variables explicativas y, por tanto, un propensity score igual o muy similar. De las distintas opciones que plantea esta técnica para realizar el emparejamiento, se ha seleccionado la del vecino más cercano y sin reemplazamiento ${ }^{5}$.

Una vez eliminadas las observaciones missing, correspondientes a las variables explicativas consideradas, la Tabla 4 muestra la probabilidad de sufrir un elevado nivel de estrés sin emparejamiento y una vez realizado el matching. En este caso, como se puede observar, ningún coeficiente ha resultado significativo individualmente, y las variables observables no consiguen explicar en absoluto la probabilidad de sufrir estrés laboral, como ponen de manifiesto los criterios pseudo-R ${ }^{2}$, LR-test observado de significación conjunta y su probabilidad.

Tabla 4

Probabilidad de declarar elevado nivel de estrés (Odds ratio) sin matching vs matching

\begin{tabular}{|c|c|c|c|c|c|c|}
\hline \multirow{3}{*}{$\begin{array}{ll} & \text { Variables } \\
\text { Estado de salud: } & \\
\text { Muy bueno } & \end{array}$} & \multicolumn{3}{|c|}{$2006 / 07$} & \multicolumn{3}{|c|}{ 2011/12 } \\
\hline & \multicolumn{2}{|c|}{ Sin matching } & Maching & \multicolumn{2}{|c|}{ Sin matching } & Maching \\
\hline & 0,6799 & $\star \star * *$ & 0,9463 & 0,6972 & *** & 0,9689 \\
\hline Bueno & 0,6660 & $\star \star \star *$ & 0,9961 & 0,7671 & $\star \star \star *$ & 1,0019 \\
\hline Enfermedad crónica & 1,2490 & $* \star *$ & 1,0364 & 1,1909 & $* \star *$ & 1,0128 \\
\hline Limitación Activ. Habitual (últimos 15 días) & 1,3475 & $\star \star \star *$ & 1,0019 & 1,2769 & $\star \star \star *$ & 0,9741 \\
\hline Accidente & 1,2597 & $\star \star * *$ & 0,9860 & 1,0409 & & 0,8511 \\
\hline \multicolumn{7}{|l|}{ Hábitos: } \\
\hline Fumador & 1,1544 & 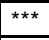 & 1,0066 & 1,1466 & $\star \star \star$ & 0,9491 \\
\hline Alcohol & 0,9935 & & 1,0170 & 0,9283 & & 1,0390 \\
\hline Horas sueño & 0,8509 & 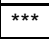 & 1,0024 & 0,8859 & $* \star *$ & 0,9779 \\
\hline Ejercicio físico & 0,9218 & * & 1,0012 & 0,8473 & 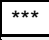 & 0,9617 \\
\hline \multicolumn{7}{|l|}{ Características personales: } \\
\hline Edad & 1,0513 & $\star \star \star *$ & 1,0249 & 1,0314 & & 0,9947 \\
\hline $\mathrm{Edad}^{2}$ & 0,9993 & 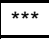 & 0,9997 & 0,9995 & * & 1,0001 \\
\hline Mujer & 1,1709 & $* \star *$ & 0,9858 & 1,1801 & $* * *$ & 0,9708 \\
\hline \multicolumn{7}{|l|}{ Responsabilidades familiares: } \\
\hline Aporta más ingresos & 1,1488 & ** & 1,0045 & 1,1363 & * & 1,0216 \\
\hline Único miembro con ingresos & 1,0186 & & 0,9700 & 0,8560 & ** & 0,9903 \\
\hline Casado & 1,1624 & ** & 0,9844 & 1,1801 & ** & 0,9187 \\
\hline Viudo & 1,3708 & * & 0,8351 & 1,7226 & $\star \star \star *$ & 0,7227 \\
\hline Separado o Divorciado & 1,2250 & ** & 0,9205 & 1,3851 & $* \star *$ & 1,0074 \\
\hline Padre o madre solo con hijos menores de 25 años & 0,7244 & ** & 1,0268 & 1,3824 & ** & 1,2564 \\
\hline Hogar con menores de 15 años & 1,1304 & & 1,0105 & 1,0059 & & 0,9398 \\
\hline Cuida solo de menores de 15 años & 0,7407 & ** & 0,9865 & 0,8230 & & 0,9703 \\
\hline Comparte cuidado de menores de 15 años & 0,8338 & * & 0,9671 & 0,8870 & & 1,0568 \\
\hline Cuida solo de discapacitados & 0,8621 & & 0,9919 & 1,2110 & & 0,9657 \\
\hline Comparte cuidado de discapacitados. & 1,2032 & & 0,9613 & 1,1422 & & 0,7004 \\
\hline \multicolumn{7}{|l|}{ Nivel educativo: } \\
\hline Educ. Secund. $2^{\mathrm{a}}$ etapa & 0,8821 & * & 0,9974 & 1,0427 & & 1,0350 \\
\hline FP superior & 1,0292 & & 1,0948 & 1,1294 & & 1,0057 \\
\hline Universitario & 1,2566 & $\star \star \star *$ & 0,9993 & 1,3072 & $\star \star \star *$ & 1,1079 \\
\hline
\end{tabular}

\footnotetext{
${ }^{5}$ Los resultados coinciden si se eligen otras opciones como la media ponderada kernel.
} 
Tabla 4 (continuación)

Probabilidad de declarar elevado nivel de estrés (Odds ratio) sin matching vs matching

\begin{tabular}{|c|c|c|c|c|c|c|c|}
\hline \multirow{3}{*}{$\begin{array}{ll} & \text { Variables } \\
\text { Relación laboral: } & \\
\text { Funcionario } & \end{array}$} & \multicolumn{3}{|c|}{$2006 / 07$} & \multicolumn{4}{|c|}{$2011 / 12$} \\
\hline & \multicolumn{2}{|c|}{ Sin matching } & Maching & \multicolumn{2}{|c|}{ Sin matching } & \multicolumn{2}{|c|}{ Maching } \\
\hline & 0,9905 & & 0,9596 & 0,8017 & * & 0,8999 & \\
\hline Indefinido & 0,9487 & & 0,9570 & 0,9439 & & 0,9296 & \\
\hline Contrato & 0,7468 & $\star * \star$ & 0,9650 & 0,8174 & ** & 0,9793 & \\
\hline \multicolumn{8}{|l|}{ Resposabilidades: } \\
\hline Muy satisfecho trabajo & 1,1941 & $\star \star \star *$ & 1,0149 & 1,1241 & ** & 1,0547 & \\
\hline Reconocimiento trabajo & 0,7520 & $\star \star \star *$ & 1,0283 & 0,7655 & $\star \star \star *$ & 0,9668 & \\
\hline Responsable de 5 a $10 \mathrm{tr}$ & 2,0636 & $\star \star \star *$ & 1,0643 & 1,6152 & $\star \star \star$ & 1,1049 & \\
\hline Responsable de 11 a $20 \mathrm{tr}$ & 1,9620 & $\star \star \star$ & 0,9225 & 1,5989 & $\star *$ & 1,1984 & \\
\hline Responsable de más 20 tr. & 1,9989 & $\star \star \star *$ & 1,0561 & 1,6574 & $\star \star \star *$ & 1,0510 & \\
\hline \multicolumn{8}{|l|}{ Jornada laboral: } \\
\hline J. partida & 1,1886 & $\star \star \star$ & 1,0422 & 1,2214 & $\star \star \star *$ & 0,9707 & \\
\hline J. reducida & 0,8303 & & 1,0723 & 0,9560 & & 1,1850 & \\
\hline J. turnos & 1,4807 & 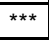 & 0,9763 & 1,2778 & 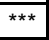 & 1,0999 & \\
\hline J. irregular o variable & 1,3591 & $* \star *$ & 0,9966 & 1,4298 & $* \star *$ & 0,9868 & \\
\hline J. otro tipo & 1,2059 & & 1,0221 & 1,3990 & * & 1,1620 & \\
\hline \multicolumn{8}{|l|}{ Comunidad Autónoma: } \\
\hline Andalucía & 1,2654 & & 1,0058 & 2,4482 & $\star \star \star *$ & 1,0484 & \\
\hline Aragón & 0,9500 & & 1,0064 & 1,6191 & $\star \star \star *$ & 1,0483 & \\
\hline Asturias & 0,9268 & & 0,9317 & 1,2289 & & 1,0090 & \\
\hline Baleares & 1,4048 & * & 1,0120 & 1,9019 & $\star \star \star$ & 1,0572 & \\
\hline Canarias & 1,4084 & * & 0,9913 & 1,8983 & $\star \star \star *$ & 1,1891 & \\
\hline Cantabria & 1,2675 & & 1,0226 & 1,5426 & $\star \star$ & 0,9655 & \\
\hline Castilla León & 1,1598 & & 1,2500 & 1,1262 & & 1,1092 & \\
\hline Castilla Mancha & 1,0458 & & 0,9249 & 1,0160 & & 1,1375 & \\
\hline Cataluña & 1,0946 & & 0,9306 & 2,3081 & $* * *$ & 0,8988 & \\
\hline Com. Valenciana & 1,0539 & & 0,9354 & 1,5360 & $\star *$ & 1,0753 & \\
\hline Galicia & 0,8832 & & 1,0688 & 1,2351 & & 1,0167 & \\
\hline Madrid & 1,1231 & & 0,9522 & 1,7606 & $\star \star \star *$ & 1,0301 & \\
\hline Murcia & 1,0304 & & 1,0344 & 2,1956 & $\star \star \star$ & 1,0056 & \\
\hline Navarra & 0,7342 & $\star *$ & 1,0436 & 0,9066 & & 0,8825 & \\
\hline País Vasco & 1,0270 & & 0,9062 & 1,3365 & * & 1,1028 & \\
\hline Rioja & 1,7428 & ** & 1,0931 & 1,2399 & & 0,9569 & \\
\hline Ceuta y Melilla & 2,2120 & 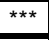 & 0,9717 & 2,1244 & 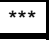 & 0,9765 & \\
\hline $\mathbf{N}$ & 12608 & & 5604 & 8318 & & 4372 & \\
\hline Pseudo $\mathrm{R}^{2}$ & 0,0466 & & 0,0018 & 0,0436 & & 0,0042 & \\
\hline LR test (chi-square) & 622,77 & 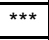 & 13,89 & 417,76 & $\star \star \star *$ & 25,34 & \\
\hline Prob (chi-square) & 0,000 & & 1,000 & 0,000 & & 1,000 & \\
\hline
\end{tabular}

Fuente: Estimación propia a partir de ENS 2006/07 y 2011/12.

Otra forma gráfica de comprobar que el emparejamiento es correcto es representar los porcentajes de sesgos estandarizados de cada una de las variables observables (Figura 1) y observar su reducción cuando se aplica el matching. Otra opción es comparar las funciones de densidad del propensity score para los dos grupos de trabajadores (Figura 2). 
Figura 1

Porcentaje de sesgos estandarizados

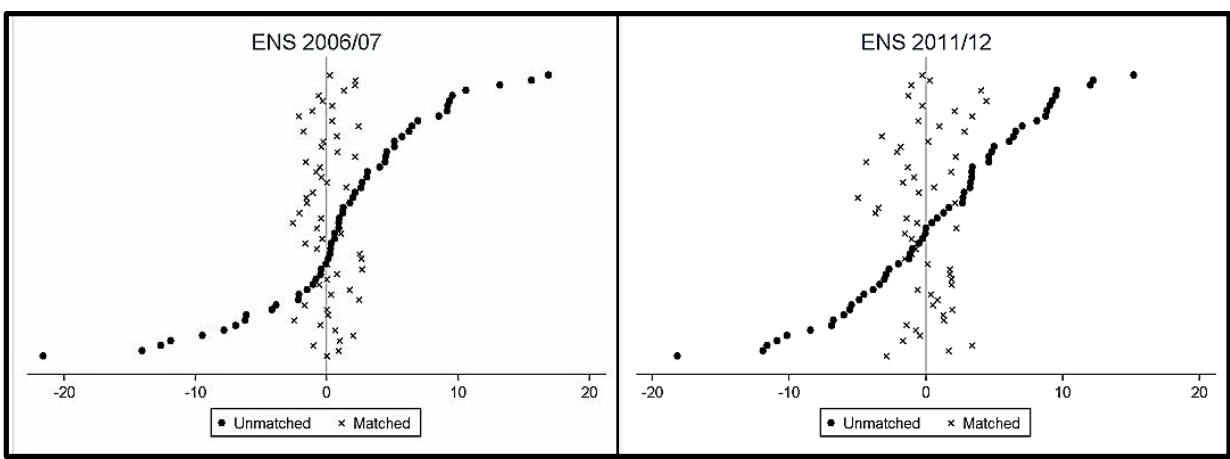

Fuente: Elaboración propia a partir de ENS 2006/07 y 2011/12.

Figura 2

Funciones de densidad del propensity score

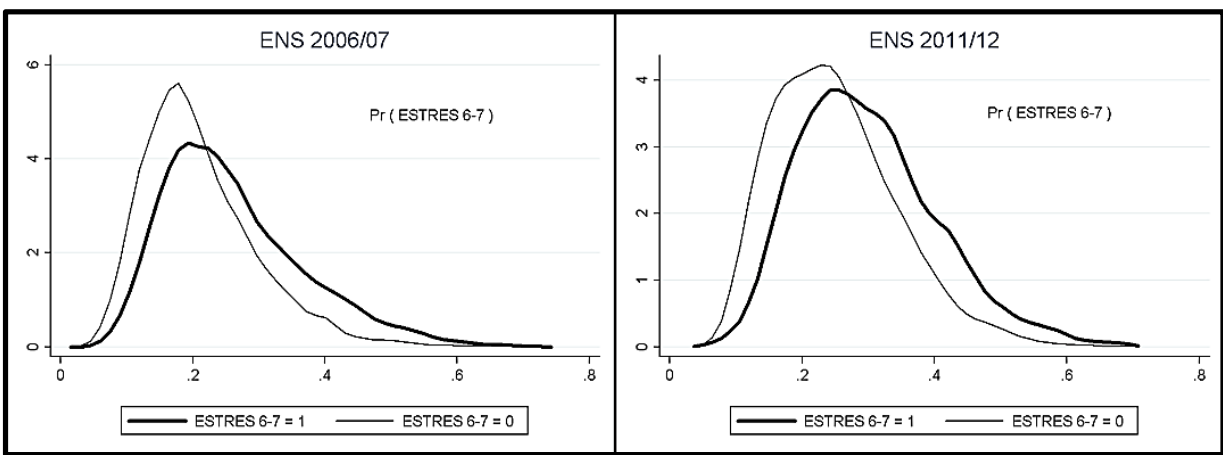

Fuente: Elaboración propia a partir de ENS 2006/07 y 2011/12.

Una vez seleccionadas las muestras de individuos comparables (2802 estresados y 2802 no estresados de la ENS 2006/07, y 2186 en cada grupo de la ENS 2011/12), el siguiente paso para cuantificar las diferencias en el número de consultas médicas atribuibles al estrés laboral consiste en estimar el número de visitas médicas mediante modelos binomial negativo, incluyendo, además del estrés, las mismas variables que se han considerado para calcular el propensity score y realizar el emparejamiento.

La Tabla 5 sólo presenta los coeficientes estimados de la variable representativa de un elevado nivel de estrés laboral, para los tres servicios sanitarios analizados en los dos periodos temporales. Los resultados confirman el hecho de que no existen diferencias en el número de visitas al especialista entre trabajadores que perciben mayores niveles de estrés laboral y aquellos que no sopor- 
tan tal riesgo, tanto en 2006/07 y 2011/12. Esta conclusión puede estar motivada por varios hechos: 1) El corto periodo de observación de la ENS, ya que sólo se refiere a las últimas cuatro semanas, cuando el tiempo de espera para recibir estas consultas es mucho más amplio de forma generalizada en todas las CCAA; 2) La propia organización asistencial del Sistema Nacional de Salud español, puesto que el médico de familia actúa como puerta de entrada al sistema y es necesaria su prescripción para poder acudir al siguiente nivel asistencial (consultas de especialidades), resolviéndose muchos de los problemas de salud en atención primaria; y 3) Las prestaciones de salud laboral se ejercen a partir de las estructuras de salud pública de las Administraciones y de la infraestructura de atención primaria del Sistema Nacional de Salud.

Con respecto al médico general, en el período 2006/07 se puede afirmar que los ocupados, que cuantifican su nivel de estrés con un 6 o un 7, consultan con el médico general en las últimas 4 semanas, por término medio, 1,134 veces más que los trabajadores no estresados ${ }^{6}$. En cambio, en 2011/12, y contrariamente a lo esperado, por las razones apuntadas de la organización del sistema, porque un mayor porcentaje de personas declaran niveles elevados de estrés, unido a unas condiciones económicas y laborales adversas en España, no se han encontrado diferencias significativas en la utilización del médico general entre los dos grupos de trabajadores.

Esta conclusión coincide con las notas de prensa publicadas por el Instituto Nacional de Estadística, en el sentido de que en el periodo transcurrido entre las dos encuestas, el estado de salud de la población, en general, ha mejorado y la utilización de servicios sanitarios ha descendido. Esta conclusión se obtiene sea cual sea la relación con la actividad económica, por lo que resulta llamativo. Obviamente, al no tratarse de la misma muestra de individuos observados en los dos períodos de tiempo no pueden extraerse conclusiones, pero quizás pueda atribuirse a que con las elevadas tasas de paro que sufre España en el periodo de la encuesta, tener trabajo y conservarlo ha pasado a ser la cuestión prioritaria. Incluso al establecer comparaciones con otros grupos de población, la salud ha descendido en el orden de preocupación ${ }^{7}$.

\footnotetext{
${ }^{6}$ Dichos valores corresponden a incidence-rate ratios. IRR $=e^{\hat{\beta}_{j}}$

${ }^{7}$ Otra posible explicación estaría basada, de nuevo, en la situación de crisis económica tan profunda como la atravesada en España en 2011/12 y en el hecho de que nuestra medida de estrés sea autopercibida. Podría ocurrir que, en momentos bajos del ciclo económico, los trabajadores declaren mayores niveles de estrés de lo que lo hubieran hecho sin crisis económica, como forma de dar más valor a su trabajo, del que muchos otros carecen (sesgo de deseabilidad social, véase nota a pie de página 2). El hecho de que declaren mayores niveles de estrés que los que les corresponderían según sus características observables podría estar afectando a las estimaciones de utilización de asistencia sanitaria.
} 
Tabla 5

Coeficientes del estrés en los modelos de utilización de asistencia sanitaria

\begin{tabular}{|l|c|c|c|c|}
\hline & \multicolumn{2}{|c|}{$2006 / 07$} & \multicolumn{2}{c|}{$2011 / 12$} \\
\hline Médico General & 0,1262 & $\star \star \star$ & -0.0333 & \\
\hline Médico Especialista & -0.0223 & & 0,0811 & \\
\hline Médico Urgencias & 0,1506 & $\star \star \star$ & 0,1569 & $* \star \star$ \\
\hline
\end{tabular}

Fuente: Estimación propia a partir de ENS 2006/07 y 2011/12.

En cuanto a la utilización de las urgencias hospitalarias, sí resultan significativas las diferencias en utilización por parte de los trabajadores que están sometidos a un elevado nivel de estrés, incluso son de la misma magnitud en los dos años. Concretamente, acuden, por término medio, 1,16 veces más al año a los servicios de urgencias hospitalarias. Este resultado también podría estar relacionado con la disminución de las visitas al médico general y el efecto de las condiciones económicas y laborales, en el sentido, de que para evitar la petición de días u horas libres para acudir al médico general, o la petición de bajas laborales, cabría pensar que la disponibilidad horaria de los servicios de urgencias pudiese ser importante a la hora de elegir dicho servicio, a pesar de que el estrés no es un problema que se trate de forma adecuada en una sala de urgencias hospitalarias con largos tiempos de espera para ser atendido, a no ser que sean casos graves que hayan derivado en crisis de ansiedad, dificultades para respirar o problemas cardíacos.

Dado que con los modelos estimados se ha intentado controlar por las características más relevantes para la explicación de la utilización de asistencia sanitaria, las diferencias en el número de consultas anteriormente indicadas pueden ser atribuibles exclusivamente al estrés laboral. Con objeto de cuantificar dicho diferencial estimado $\left(\Delta \hat{U}_{E}\right)$, es preciso obtener el número medio de visitas de los “no estresados” $\left(\bar{U}_{N E}\right)$ y aplicar la fórmula:

$$
\Delta \hat{U}_{E}=\left(e^{\hat{\beta}_{E}}-1\right) \cdot \bar{U}_{N E}
$$

cuyos resultados se exponen en la Tabla $6^{8}$.

Al comparar estas cifras con las obtenidas calculando el número medio de visitas entre los dos grupos de trabajadores (Tabla 3) se observa como la influencia del estrés laboral sobre las visitas estaba "contaminada” por otros factores que también inciden en el estrés laboral.

\footnotetext{
${ }^{8}$ Únicamente se exponen los de aquellos servicios que mostraron diferencias significativas respecto al estrés. Todos los cálculos posteriores sólo harán referencia a estos casos.
} 
Tabla 6

Diferencial en el número medio de visitas atribuible exclusivamente al estrés laboral

\begin{tabular}{|l|c|c|c|c|}
\hline & \multicolumn{2}{|c|}{ Diferencial 06/07 } & \multicolumn{2}{c|}{ Diferencial 11/12 } \\
\hline & $\bar{U}_{N E}$ & $\Delta \hat{U}_{E}$ & $\bar{U}_{N E}$ & $\Delta \hat{U}_{E}$ \\
\hline Médico General (mes) & 0,3474 & 0,0467 & - & - \\
\hline Médico Urgencias (12 meses) & 0,5011 & 0,0814 & 0,4416 & 0,0750 \\
\hline
\end{tabular}

Fuente: Estimación propia a partir de ENS 2006/07 y 2011/12.

Con objeto de homogeneizar los datos y efectuar comparaciones referidas al mismo periodo temporal, es preciso extrapolar los resultados relativos a la utilización del médico de familia durante las últimas cuatro semanas (mes), a términos anuales, multiplicando las cifras anteriores por 12. Dicha operación no está basada en que la utilización de servicios sanitarios de los individuos entrevistados en ese período se vaya a repitir en los doce meses del año, puesto que no tendría ningún sentido, sino en que la muestra con la que se ha realizado la estimación es representativa de la población y reproduce la estructura de utilización durante un mes cualquiera. Por tanto, si se seleccionara otra muestra referida a otro mes, los resultados deberían ser muy similares.

Por último, antes de evaluar el coste monetario, hay que tratar de cuantificar el número de visitas a los servicios sanitarios analizados para toda la población española y deducir qué parte de esa cantidad se debe exclusivamente al estrés en el trabajo. Para ello, se utiliza la cualidad de representatividad de la muestra para elevar los resultados a nivel nacional, teniendo en cuenta los factores de ponderación o pesos de cada observación.

En la tercera columna de la Tabla 7 se muestra el total de visitas al médico general y urgencias realizadas por la población ocupada debidas a múltiples causas. Para calcular qué parte de esa utilización total de la población se debe exclusivamente al estrés laboral $\left(\Delta \hat{U} T_{E}\right)$, se debe recurrir a la información sobre diferenciales en el número medio de visitas recogidos en la Tabla 6 . Además de la estimación puntual, se ha calculado un intervalo de confianza al 95 por ciento. En este caso, puesto que dichos diferenciales tienen una distribución log-normal, las esperanzas y varianzas se han obtenido a partir de las siguientes fórmulas:

$$
\begin{gathered}
E\left(\Delta \hat{U} T_{E}\right)=\operatorname{Pob}_{E} * \bar{U}_{N E} *\left(e^{\hat{\beta}_{E}+\frac{\hat{\sigma}_{\hat{\beta}_{E}}^{2}}{2}}-1\right) \\
\operatorname{Var}\left(\Delta \hat{U} T_{E}\right)=\operatorname{Pob}_{E}^{2} * \bar{U}_{N E}^{2} *\left(e^{\hat{\sigma}_{\hat{\beta}_{E}}^{2}}-1\right) *\left(e^{2 * \hat{\beta}_{E}+\hat{\sigma}_{\hat{\beta_{E}}}^{2}}\right)
\end{gathered}
$$


Tabla 7

Estimación del número de visitas médicas anuales en España

\begin{tabular}{|c|l|c|c|c|c|}
\hline & & & \multicolumn{2}{|c|}{ Número de visitas por estrés laboral } \\
\hline \multirow{2}{*}{ Año } & \multicolumn{1}{|c|}{ Servicio } & $\begin{array}{c}\text { Total } \\
\text { visitas }\end{array}$ & $\begin{array}{c}\text { Estimación } \\
\text { puntual }\end{array}$ & \multicolumn{2}{|c|}{$\begin{array}{c}\text { Intervalo confianza } \\
\text { (95\%) }\end{array}$} \\
\hline 2006 & M. General & 67.609 .836 & 2.242 .948 & 741.286 & 3.790 .171 \\
\cline { 2 - 6 } 2007 & M. Urgencias & 8.130 .164 & 325.592 & 141.195 & 515.566 \\
\hline 2011 & M. Urgencias & 6.715 .842 & 342.891 & 119.403 & 574.499 \\
2012 & & & & &
\end{tabular}

Fuente: Elaboración propia a partir de ENS 2006/07 y 2011/12.

Como se puede observar, las visitas al médico general en 2006/07, motivadas por problemas de estrés laboral, superan los 2,2 millones. La cifra de urgencias debidas al estrés es sensiblemente menor, dada la configuración del sistema y las características del problema de salud que estamos considerando, sin embargo, es similar en los dos años, a pesar de que el número total de visitas a urgencias ha descendido de 8,13 a 6,7 millones. En términos porcentuales, las visitas al médico general por estrés suponen en torno al 3,3 por ciento del total de las visitas realizadas por el colectivo de trabajadores. Las visitas a urgencias representan el 4 y el 5,1 por ciento en 2006/07 y 2011/12, respectivamente.

Dado que el estrés no está considerado aún como una enfermedad profesional, con las estimaciones realizadas en este trabajo se pone de manifiesto que un importante volumen de servicios sanitarios (2,5 millones de visitas, aproximadamente, en 2006/07 y 343.000 visitas en 2011/12) y su coste lo está asumiendo el Sistema Nacional de Salud y, por tanto, toda la sociedad, cuando deberían ser las Mutuas de Accidentes o las empresas quienes soportasen dicha factura.

\section{EVALUACIÓN MONETARIA DEL COSTE SANITARIO ASOCIADO AL ESTRÉS LABORAL}

Precisamente, la mayor dificultad a la hora de cuantificar el coste sanitario asociado al estrés laboral radica en ese hecho, es decir, no estar contemplado como enfermedad laboral o enfermedad del trabajo en el catálogo establecido por la normativa laboral vigente en España. En general, los trabajadores severamente afectados por estrés que acuden a su médico de atención primaria son dados de baja por enfermedad común y, en la mayor parte de los casos, por depresión. El hecho de que el estrés no sea la única causa de esas enfermedades comunes imposibilita utilizar algunas de las bases de datos sanitarias del Ministerio de Sanidad, Servicios Sociales e Igualdad, como apoyo en la tarea de evaluar el coste sanitario derivado de aquella dolencia.

El trabajo de García-Gómez et al., (2011), sobre enfermedades mentales derivadas del trabajo en España, trata de estimar el coste sanitario directo de las 
mismas, pero sólo recopila información del Conjunto Mínimo Básico de Datos al alta hospitalaria, del Sistema de Información de los Establecimientos con Régimen de Internado y del Registro de altas de Atención Ambulatoria Especializada, es decir, sólo de aquellos casos que han requerido hospitalización con o sin ingreso. Tampoco puede imputar el coste debido al estrés, puesto que las dos últimas fuentes de información no recogen la categoría diagnóstica (depresión, ansiedad, estrés, etc.).

En este trabajo se propone realizar una evaluación económica de la utilización debida al estrés, semejante a la diseñada por la Administración para los servicios sanitarios prestados a trabajadores que han sufrido accidentes de trabajo o enfermedades profesionales.

El Real Decreto 1030/2006 de 15 de septiembre, relativo a la cartera de servicios comunes del Sistema Nacional de Salud, establece en su Anexo IX, apartado 3, que los servicios públicos de salud reclamarán a los terceros obligados al pago el importe de las atenciones o prestaciones sanitarias facilitadas directamente a las personas, en el supuesto de accidentes de trabajo o enfermedades profesionales a cargo de las Mutuas Patronales de Accidentes de Trabajo, del Instituto Nacional de la Seguridad Social o del Instituto Social de la Marina.

La Orden TASS/131/2006 de 26 de enero, en su capítulo II, fija el procedimiento de pago a los Servicios Públicos de Salud del coste de la asistencia sanitaria derivada de contingencias profesionales. En su artículo 5 se especifica que los Servicios Públicos de Salud de las Comunidades Autónomas remitirán mensualmente a las Direcciones Provinciales de las entidades gestoras, una certificación del coste de las prestaciones sanitarias, farmacéuticas y recuperadoras, dispensadas en el mes anterior, junto con el detalle de las prestaciones dispensadas a cada beneficiario. Las Direcciones Provinciales comprobarán que los gastos corresponden a trabajadores protegidos, y que los importes de las prestaciones dispensadas coinciden con las cuantías de las tarifas oficiales aprobadas y publicadas por cada Comunidad Autónoma.

Dichas tarifas o precios públicos son fijados por las distintas Consejerías de Salud o Sanidad de las Comunidades Autónomas, bajo la fórmula de Órdenes, Resoluciones, Decretos o Acuerdos. Como consecuencia, para la valoración económica de las diferencias en utilización de asistencia sanitaria motivadas por estrés laboral, se han aplicado las tarifas o precios públicos vigentes para cada una de las CCAA en los años 2006 y 2012, correspondientes a las consultas de atención primaria y urgencias hospitalarias. El coste para las ciudades de Ceuta y Melilla las establece el Ministerio de Sanidad desde que se culminó el proceso de transferencias en materia sanitaria en diciembre de $2001^{9}$.

\footnotetext{
${ }^{9}$ La Comunidad de Castilla-León, desde que recibió las citadas transferencias, no había fijado precios públicos para el año 2006 por lo que se han valorado con el precio fijado por el Ministe-
} 
En la tercera columna de la Tabla 8 se muestra el coste total por asistencia sanitaria calculado con tales precios públicos. En concreto, la cifra se corresponde con el producto del número de visitas realizadas a cada servicio en un año por el precio público de dicho servicio en la Comunidad Autónoma en la que resida el trabajador. Al sumar, de manera ponderada (utilizando los pesos muestrales), tales cantidades para todos los individuos que componen la muestra, se llega al importe total de la factura asociada a la utilización de asistencia sanitaria por parte de los trabajadores en España. La cifra de coste total de las visitas al médico general y urgencias hospitalarias en 2006/07 asciende a 4210 millones $€$. Para 2011/12, los costes de las urgencias oscilan en torno a los 1121 millones $€$.

De nuevo, las cifras de coste total por utilización de asistencia sanitaria atribuible al estrés laboral se obtienen a partir de las estimaciones sobre diferenciales en el número medio de visitas anuales recogidas en la Tabla 6. En concreto, para cada ocupado sometido a riesgo de estrés encuestado en la ENS, se ha calculado el producto del diferencial correspondiente por el precio público del servicio sanitario en su Comunidad Autónoma. Dichas cantidades se han agregado teniendo en cuenta, de nuevo, el factor de elevación a la población que corresponde a cada observación muestral, hasta llegar a una cifra global indicativa del coste sanitario asociado al estrés laboral. La estimación puntual se complementa con la construcción de un intervalo de confianza a partir de las fórmulas [4] y [5]. Finalmente, el coste total de utilización de las visitas al médico general y a las urgencias hospitalarias atribuible al estrés laboral se eleva a los 145,26 millones $€$ en 2006/07 y a 55,17 millones $€$ en 2011/12.

Tabla 8

Estimación del coste monetario del número de visitas médicas anuales en España

\begin{tabular}{|c|l|r|r|r|r|}
\hline & & & \multicolumn{2}{|c|}{ Coste por estrés laboral } \\
\hline \multirow{2}{*}{ Año } & Servicio & $\begin{array}{c}\text { Coste total } \\
\text { Visitas } \\
\text { (millones } € \text { ) }\end{array}$ & $\begin{array}{c}\text { Estimación } \\
\text { Puntual } \\
\text { (millones } € \text { ) }\end{array}$ & \multicolumn{2}{|c|}{$\begin{array}{c}\text { Intervalo } \\
\text { confianza (95\%) } \\
\text { (millones } € \text { ) }\end{array}$} \\
\hline \multirow{2}{*}{2006} & M.General & 3044,400 & 99,979 & 32,711 & 167,250 \\
\cline { 2 - 6 } 2007 & M. Urgencias & 1165,750 & 45,283 & 19,470 & 71,096 \\
\cline { 2 - 6 } & Total & 4210,150 & 145,262 & 52,181 & 238,346 \\
\hline 2011 & M. Urgencias & 1121,830 & 55,175 & 18,989 & 91,362 \\
\hline 2012 & & & & & \\
\hline
\end{tabular}

Fuente: Elaboración propia a partir de ENS 2006/07 y 2011/12.

rio para Ceuta y Melilla en dicho año. Por otra parte, Aragón estableció hasta 2006 aplicar las tarifas aprobadas por el Instituto Nacional de la Salud, en la Resolución de 26 de diciembre de 2001 (BOE $\mathrm{n}^{\circ}$ 5, 05/01/2002), actualizadas según el IPC interanual de la subclase "servicios hospitalarios" correspondiente al mes de diciembre inmediatamente anterior. 
A pesar de la relevancia de estas cifras, resultan menores que las obtenidas por otros autores. Perkins (1994) presenta investigaciones que muestran que entre el $60 \%$ y el $90 \%$ de todas las visitas al médico están relacionadas con el estrés. Estudios en economía de la salud, como el estudio HERO (1998), concluyen que los gastos en cuidados de salud de trabajadores que reportan altos niveles de estrés son un $50 \%$ más altos que los de aquéllos que no tienen tal nivel de estrés.

\section{CONCLUSIONES}

Aunque el problema del estrés laboral es percibido como de gran magnitud, no han sido muchos los intentos por evaluar en términos monetarios el coste que conlleva la existencia de entornos laborales estresantes para la sociedad en general. Tomando como referencia el caso español, ése ha sido, precisamente, el objetivo fundamental que ha perseguido esta investigación. Más en concreto, se ha pretendido obtener una visión cuantitativa de la importancia de la existencia de un entorno de trabajo libre de estrés y documentar los potenciales beneficios de su prevención eficiente. El centro de atención ha recaído en los costes médicos originados por la existencia de entornos de trabajo estresantes y que son asumidos por toda la sociedad.

La estimación de modelos econométricos para el número de consultas al médico general y visitas a urgencias han permitido confirmar la hipótesis de que en 2006 los trabajadores sometidos a situación de estrés tenían una mayor propensión a la utilización de los servicios sanitarios. En cambio, y contrariamente a lo esperado, en 2011 los resultados concluyen que estos trabajadores únicamente realizan un mayor uso de las visitas a urgencias, siendo tal diferencia de la misma magnitud que la obtenida en 2006, a pesar de que la cifra total de urgencias ha descendido. Podría pensarse que esto es un logro porque en el periodo transcurrido se han diseñado políticas adecuadas para mejorar la calidad de vida del trabajo, que han reducido el nivel de estrés laboral y, por tanto, la necesidad de acudir al médico. Sin embargo, la grave situación económica puede haber provocado cambios en la percepción y las prioridades de los trabajadores y de la población en general, que expliquen la mejora del estado de salud autopercibido y la disminución de la utilización al médico general.

Una de las ventajas de la metodología adoptada en este trabajo radica en la utilización de una encuesta representativa a nivel nacional de la población que incluye información sobre estrés. Esto ha permitido examinar la relación del estrés con la demanda de servicios sanitarios y extrapolar los resultados a nivel nacional. A pesar de ello, la ENS también presenta algunas limitaciones como el reducido periodo de observación de las consultas al médico general y al especialista o que la valoración del estrés está basada en la propia percepción del trabajador y no en un diagnóstico clínico. 
Dados los resultados obtenidos en esta investigación sobre los costes asociados al estrés laboral, sería recomendable que, tanto desde el lado de la Administración como de las empresas u organizaciones, se aborde este tema con mayor seriedad, destinando el tiempo y los recursos necesarios para su prevención y control. Las empresas deberían desarrollar claras políticas anti-estrés, contando para ello con el aliento de los poderes públicos. Por su parte, los sindicatos deben desempeñar un papel principal en la concienciación sobre los efectos perniciosos del estrés, siendo aún más activos, conminando a las organizaciones a que monitoricen sus actividades y procedimientos anti-estrés por medio de la realización de auditorías habituales y asegurándose de que se proporcionan recursos adecuados para abordar el problema. También resulta necesaria una investigación más profunda sobre las implicaciones a largo plazo del estrés sobre los individuos, para poder establecer cómo conseguir una rehabilitación exitosa de los trabajadores expuestos. Por último, para seguir investigando en estos temas sería imprescindible diseñar bases de datos a nivel nacional más específicas del entorno laboral que también aborden aspectos sobre la salud y la utilización de servicios sanitarios.

En consecuencia, el estrés laboral no es sólo un problema global y creciente, sino también un problema tan destructivo para el bienestar de los trabajadores como costoso para las empresas y para la sociedad, en general. Un país como España, que afronta en estos momentos déficits de productividad significativos, no puede permitirse "negatividades" de esta índole.

\section{REFERENCIAS BIBLIOGRÁFICAS}

AKERSTEDT, T., KNUTSSON, A., WESTERHOLM, P., THEORELL, T., ALFREDSSON, L., KECKLUND, G. (2002). "Sleep disturbances, work stress and work hours: a cross-sectional study". Journal of Psychosomatic Research, 53, pp. 741-748.

AZAGBA, S., SHARAF, M. (2011). "Psychosocial working conditions and the utilization of health care services". BMC Public Health, 11, pp. 642-648.

BENEDITTIS, G.D., LORENZETTI, A. ( 1992). "The role of stressful life events in the persistence of primary headache: Majors events vs. daily hassles". Pain, 51, pp. 35-42.

BROSSCHOT, J.F., BENSCHOP, R.J., GODAERT, G., OLFF, M., DESMET, M., HEIJNEN, C.J., BALLIEUX, R.E. (1994). "Influence of life stress on immunological reactivity to mild psychological stress". Psychosomatic Medicine, 56, pp. 216-224. 
CAMERON, A.C.; TRIVEDI, P.K.; MILNE, F. y PIGGOTT, J. (1988). "A microeconometric model of the demand for health care and health insurance in Australia". Review of Economic Studies, LV, pp. 85-106.

CHANDOLA, Y., BRITTON, A., BRUNNER, E., HEMINGWAY, H., MALIK, M., KUMARI, M., BADRICK, E., KIVIMAKI, M., MARMOT, M. (2008). "Work stress and coronary heart disease: what are the mechanisms?". European Heart Journal, 29, pp. 640-648.

COCHRAN, W.G., RUBIN, D.B. (1973). "Controlling bias in observational studies: A review". Sankhya, Series. A, 35, pp. 417-446.

COOPER, ML., RUSSELL, M., FRONE, MR. (1990). "Work stress and alcohol effects: a test of stress-induced drinking". Journal of Health and Social Behaviour 31(3), pp. 260-276.

COX, T. (1993). Stress Research and Stress Management: Putting Theory to Work. HSE Contract Research Report, NO 61. London: HMSO.

DANO, A.M. (2005). "Road injuries and long-run effects on income and employment". Health Economics, 14, pp. 955-970.

DI MARTINO, V. (1992). Occupational stress: a preventive approach, en OIT Conditions of Work Digest, 11/2, 3-21. Geneva: OIT.

EASHW- European Agency for Safety and Health at Work (2007). Expert forecast on emerging psychosocial risks related to occupational safety and health, Luxemburgo: Office for Official Publications of the European Communities.

EC-DGESA (1999). Guidance on work-related stress. Spice of life or kiss of death?, Luxemburgo: Office for Official Publications of the European Communities.

FREEMAN, R. B. (1978). "Job satisfaction as an economic variable". American Economic Review, 68, pp. 135-141.

FRENCH, M. T. y DUNLAP, L. (1998). "Compensating wage differentials for job stress". Applied Economics, 30, pp. 1067-1075.

FROLICH, M., HESMATI, A., LECHNER, M. (2004). "A microeconometric evaluation of rehabilitation of long-term sickness in Sweden". Journal of Applied Econometrics, 19, pp. 375-396.

GAMERO, C. (2010a). "Análisis económico de los determinantes del estrés laboral en España". Estadística Española, 52, 175, pp. 393-417.

GAMERO, C. (2010b). "Assessing the cost of lost working days associated with job stress: a proposal for Spain". Estudios de Economía Aplicada, 28 (3), pp. 1-20.

GARCíA-GÓMEZ, M., OLIVA, J., URBANOS, R., DE LA CRUZ, O., CASTAÑEDA, R. (2012). Costes laborales de las enfermedades mentales atribuibles al trabajo en España 2010. Madrid: UGT-CEC.

GARCíA-GÓMEZ, M., CASTAÑEDA, R., URBANOS, R., DE LA CRUZ, O., LÓPEZ, P. (2011). Enfermedades mentales derivadas del trabajo en España 
y su coste sanitario directo en 2010. Madrid: UGT-CEC.

GARCÍA-GÓMEZ, P. (2011). "Institutions, health shocks and labour market outcomes across Europe". Journal of Health Economics, 30, pp. 200-213.

GARCÍA-GÓMEZ, P. LOPEZ-NICOLÁS, A. (2006). "Health shocks, employment and income in the Spanish labour market". Health Economics, 15, pp. 997-1009.

GORTMAKER, SL., ECKENRODE, J., GORE, S. (1982). "Stress and utilization of health services: a time series and cross-sectional analysis". Journal of Health and Social Behavior, 23, pp. 24-38.

GROOT, W. y MAASSEN VAN DEN BRINK, H. (1999). "The price of stress". Journal of Economic Psychology, 20, pp. 83-103.

GROSSMAN, M. (1972a). The demand for health: a theoretical and empirical investigation, New York: National Bureau of Economic Research, Columbia University Press.

GROSSMAN, M. (1972b). "On the concept of health capital and the demand for health". Journal of Political Economy, 80, pp. 223-255.

HERO-Health Enhancement Research Organization Research Committee (1998). "The relationship between modifiable health risks and health care expenditures: an analysis of the multi-employer HERO health risk and cost database". Journal of Occupational and Environmental Medicine, 40 (10), pp. 843-854.

KARASEK, R., THEORELL, T. (1990). Healthy work:stress, productivity, and the reconstruction of working life. New York: Basic Books.

KOUVONEN, A., VAHTERA, J., OKSANEN, T., PENTTI, J., VÄÄNÄNEN, AK., HEPONIEMI, T., SALO, P., VIRTANEN, M., KIVIMÄKI, M. (2013). "Chronic workplace stress and insufficient physical activity: a cohort study". Occupational Environmental Medicine, 70(1), pp. 3-8.

KRAMER, J.R., LEDOLTER, J., MANOS, G.N., BAYLESS, M.L. (2000). "StresS and metabolic control in diabetes mellitus: Methodological issues and an illustrative analysis". Annals of Behavioral Medicine, 22, pp. 17-28.

LECHNER, M., VAZQUEZ-ALVAREZ, R. (2004). The effect of disability on labour outcomes in Germany: evidence from matching. Discussion Paper No. 4223. Centre for Economic Policy Research, London.

LEONTARIDI, R.M. y WARD, M.E. (2002). "Work-Related Stress, Quitting Intentions and Absenteeism". IZA Discussion Paper No. 493.

MACFADYEN, A. J., MACFADYEN, H. W. Y PRINCE, N. J. (1996). "Economic stress and psychological well-being: an economic psychology framework". Journal of Economic Psychology, 17, 3, pp. 291-312.

MECHANIC, D. (1979). "Correlates of physician utilization: Why do major multivariate studies of physician utilization find trivial psychosocial and organizational effects?". Journal of Health and Social Behavior, 20, pp. 387-396. 
PERKINS, A. (1994). "Saving money by reducing stress". Harvard Business Review, 72(6), 12.

ROSENBAUM, P., RUBIN, D.B. (1983). "The central role of the propensity score in observational studies for causal effects". Biometrika, 70: 41-55.

ROSENGREN, A., HAWKEN, S., OUNPUU, S., SLIWA, K., ZUBAID, M., ALMAHMEED, WA., BLACKETT, K.N., SITTHI, C., SATO, H., YUSUF, S. (2004). "Association of psychosocial risk factors with risk of acute myocardial infarction in 11119 cases and 13648 controls from 52 countries: case-control study". Lancet, 364, pp. 953-962.

SCHIMDT, A., NEUMANN, M., WIRTZ, M., ERNSTMANN, N., STARATSCHEK-JOX, A., STOELBEN, E., WOLF, J., PFAFF, H. (2010). "The influence of occupational stress factors on the nicotine dependence: a cross sectional study". Tobacco Induced Diseases, 8:6.

SORBI, M.J., MAASEN, G.H., SPIERINGS, E. (1996). "A time-series analysis of daily hassles and mood changes in the 3 days before the migraine attack". Behavioral Medicine, 22, pp. 102-113.

TURNER-COBB, J.M., STEPTOE, A. (1996). "Psychosocial stress and susceptibility to upper respiratory tract illness in an adult population sample". Psychosomatic Medicine, 58, pp. 404-412.

URBANOS, R. (2000). "Desigualdades sociosanitarias y efectividad potencial de las políticas públicas: un estudio aplicado con datos españoles". Hacienda Pública Española, 154 (3), pp. 217-238.

VERA-HERNÁNDEZ, A.M. (1999). "Duplicate coverage and demand for health care. The case of Catalonia". Health Economics, 8, pp. 579-598.

ZWEIFEL, P. (1981). "Supplier-induced demand in a model of physician behaviour". En Van der Gaag, J. y Perlman, M. (eds.): Health, Economics, and Health Economics (pp. 245-267). Amsterdam: North Holland. 
